\title{
ATIVIDADE ANTIFÚNGICA DE Anadenanthera macrocarpa (Benth) Brenam SOBRE Candida krusei
}

\author{
Nabuêr Silva ${ }^{1 *}$, Gisele Silva ${ }^{2}$, Maria Tamires Espindola ${ }^{2}$ \\ Wanessa Gomes ${ }^{2}$, Rosângela Falcão ${ }^{2}$, Ivone Souza ${ }^{1}$ \\ ${ }^{1,5}$ Laboratório de Cancerologia Experimental, UFPE; ${ }^{2}$ Laboratório de Biotecnologia, UPE, Campus Garanhuns. \\ *nabuerfrancieli@gmail.com
}

\begin{abstract}
INTRODUÇÃO
$\mathrm{O}$ uso de plantas pelo homem com fins terapêuticos data desde os primórdios da civilização e os metabólitos secundários produzidos por vegetais em resposta a agressões sofridas têm se destacado por sua fundamental importância na área farmacológica, uma vez que possuem efeitos biológicos sobre várias patologias que acometem os seres humanos, sendo importantes fontes de pesquisa para determinação da farmacodinâmica na produção de fitofármacos (PEREIRA; CARDOSO, 2012).
\end{abstract}

0 aumento da resistência dos microrganismos aos fármacos usuais tem gerado preocupação refletindo em uma problemática no â mbito da saúde pública, com isso torna-se fundamental o desenvolvimento de pesquisas no intuito de buscar novas opções terapêuticas, com maior eficiência em relação as terapias já estabelecidas no mercado para o tratamento de espécies resistentes de microrganismos patogênicas. Nesse sentindo, a busca por novos agentes terapêuticos na flora de uso medicinal tem se mostrado uma via promissora. Dentre as espécies medicinais, Anadenanthera macrocarpa, conhecida vulgarmente como angico, destaca-se pelo uso contra várias doenças na medicina tradicional, como para o tratamento de tosse, bronquite, feridas externas e inflamação (MATOS, 1997).

Sobre o exposto acima, Volpato (2005) destaca que a atividade antimicrobiana é uma das mais pesquisadas em plantas devido aos problemas do uso irracional de antibióticos tradicionais, causando estirpes resistentes aos mesmos, quanto as atividades farmacoló gicas atribuídas a esses organismos.

Dentre as patologias clínicas causadas por microrganismos, cita-se a candidíase, enfermidade oportunista causada por fungos do gê nero Candida. Esta caracteriza-se por lesões que podem variar de superficiais a profundas; brandas, agudas ou crônicas; envolvendo diversos sítios, tais como boca, garganta, língua, pele, couro cabeludo, genitálias, dedos, unhas e por vezes órgãos internos. A espécie Candida albicans chama atenção por sua maior associação com o desenvolvimento desta doença (BARBEDO; SGARBI, 2010).

Contudo, o perfil etiológico das espécies responsáveis pelo desenvolvimento de candidíase vem sofrendo mudanças. A incidê ncia desta doença por espécies não-albicans têm se tornado mais comum, além disso, a ocorrência de cepas resistentes entre essas espécies merece maior destaque, as quais cita-se Candida krusei (JÚNIOR; GRIGOLETO; FREGONEZI, 2011; TORTORA; FUNKE; CASE, 2012). Nessa perspectiva, Penna et al. (2001), argumentam sobre a importância do desenvolvimento de pesquisas que investiguem novos agentes antimicrobianos, em resposta ao surgimento de microrganismos resistentes e de infecções oportunistas fatais, associadas a AIDS, quimioterapia antineoplásica e transplantes.
Dessa forma, muito são os estudos que investem na descoberta de novas substâncias que apresentem efeito antimicrobiano, possibilitando o desenvolvimento de drogas mais eficientes contra espécies resistentes e prevenindo o seu surgimento (FERNANDES, 2014; LEITE et al., 2015; RODRIGUES et al., 2014; SILVA, 2011).

Nesse aspecto, o presente estudo foi desenvolvido com o objetivo de investigar o potencial antifúngico de extratos etanólicos das cascas de Anadenanthera macrocarpa contra Candida krusei com base em um estudo etnofarmacológico realizado em comunidades quilombolas situadas na área rural do município de Garanhuns-PE.

\section{MATERIAIS E MÉTODOS}

Seleção, coleta do material botânico e local de realização da pesquisa

A coleta da espécie medicinal em estudo foi feita na zona rural do município de Garanhuns-PE, em comunidades quilombolas. A espé cie foi selecionada através de informações etnobotânicas (Tabela 1) coletadas a partir de entrevista com aplicação de questionário semi-estruturado com moradores dessas comunidades sob autorizaç ão e aprovação do projeto junto ao Comitê de Ética em comprimento as exigências legais (CAAE No. 24906014.9.0000.5207).

Tabela 1. Espécie medicinal selecionada para o estudo, citada em entrevistas por moradores das comunidades quilombolas do município de Garanhuns-PE, 2014.

\begin{tabular}{lccccc}
\hline Espécie vegetal & Família & $\begin{array}{c}\text { Nome } \\
\text { local }\end{array}$ & $\begin{array}{c}\text { Indicações terapê } \\
\text { uticas }\end{array}$ & $\begin{array}{c}\text { Forma } \\
\text { de uso }\end{array}$ & $\begin{array}{c}\text { Parte } \\
\text { utilizada }\end{array}$ \\
\hline $\begin{array}{c}\text { Anadenanthera } \\
\text { macrocarpa }\end{array}$ & Anacardiaceae & Angico & $\begin{array}{c}\text { Anti-inflamatório, } \\
\text { cicatrizante }\end{array}$ & $\begin{array}{c}\text { Banho } \\
\text { de } \\
\text { assento }\end{array}$ & Cascas \\
\hline Fonte: Produção do autor (2017). & & & & & \\
\hline
\end{tabular}

Padronização do extrato etanólico

A obtenção do extrato etanólico foi realizada no Laboratório de Biotecnologia da Universidade de Pernambuco (UPE), Campus Garanhuns. Para preparação do extrato etanólico, foram utilizadas cascas secas de $A$. macrocarpa as quais permaneceram submersas em recipiente contendo etanol puro. Logo após o extrato foi filtrado e concentrado em rotaevaporador.

Linhagem utilizada

Foi utilizada a linhagem 5840 de Candida krusei. Sendo esta cedida pela Universidade Federal Rural de Pernambuco, unidade acadê mica de Garanhuns. A cepa havia sido depositada pelo Cerpe Laboratórios na Micoteca da Universidade Federal de Pernambuco, Campus Recife, anteriormente coletada de uma amostra de sangue 
via hospedeiro, seguindo as exigências legais para realização do procedimento.

\section{Avaliação da atividade antifúngica por difusão em ágar}

A atividade antifúngica do extrato etanólico de $A$. macrocarpa foi realizada de acordo com a metodologia de ágar em disco-difusão adotada por Koneman et al. (1997). Os extratos foram avaliados nas concentrações de $25 \mathrm{mg} / \mathrm{ml}, 50 \mathrm{mg} / \mathrm{ml}, 100 \mathrm{mg} / \mathrm{ml}$ com base na metodologia de Alfaia e Aumeida (2016). Para realização da té cnica de disco-difusão foram utilizados discos de papel estéreis de $6 \mathrm{~mm}$ de diâmetro impregnados com $10 \mu \mathrm{L}$ dos extratos de $A$. macrocarpa. Depois de preparados, os discos foram depositados em placas de petri contendo ágar sabouraud previamente inoculadas com suspensão microbiana. Foram preparadas suspensões de cultura, diluídas em solução salina $0,85 \%$ utilizando a escala de 0,5 de MacFarland até a obtenção de aproximadamente de $1 \times 10^{6}$ a 5 $X 10^{6}$ células por $\mathrm{mL}$ de leveduras (NCCLS, 2002). Os testes foram realizados em triplicata, sendo as placas incubadas a $35{ }^{\circ} \mathrm{C}$ por aproximadamente 48 horas. Como padrão positivo foram utilizados os antifúngicos comerciais miconazol e nistatina, enquanto padrão negativo utilizou-se o diluente dimetilsulfóxido (DMSO).

Os resultados foram obtidos por meio da medição em milímetros dos halos de inibição formados, com auxílio de uma régua milimetrada.

\section{RESULTADOS E DISCUSSÃO}

O bioensaio de difusão em ágar pelo método de disco, mostrou que o extrato etanólico das cascas de Anadenanthera macrocarpa não foi capaz de inibir o crescimento microbiano (Tabela 2), em ambas as concentrações testes, demonstrando desta maneira que o microrganismo padrão apresentou resistência ao extrato nas condiç ões experimentais avaliadas.

Tabela 2. Medidas dos diâmetros dos halos de inibição de crescimento microbiano $(\mathrm{mm})$ obtidas pela metodologia de difusão em ágar por disco utilizando extratos etanólicos de Anadenanthera macrocarpa frente Candida krusei.

\begin{tabular}{l|c|c|c}
\multicolumn{3}{c}{ Anadenanthera macrocarpa frente Candida krusei. } \\
\hline \multirow{3}{*}{ Planta } & \multicolumn{3}{|c}{ Concentração do extrato etanólico $(\mathbf{m g})$} \\
\cline { 2 - 4 } & $25 \mathrm{mg}$ & $50 \mathrm{mg}$ & $100 \mathrm{mg}$ \\
\cline { 2 - 4 } & $\begin{array}{c}\text { Média da zona de } \\
\text { inibição }\end{array}$ & $\begin{array}{c}\text { Média da zona de } \\
\text { inibição }\end{array}$ & $\begin{array}{c}\text { Média da zona de } \\
\text { inibição }\end{array}$ \\
\hline Angico & - & - & - \\
\hline
\end{tabular}

(-): ausência de zona de inibição.

Entretanto, outros trabalhos na literatura, comprovam o potencial antimicrobiano desta espécie como o de Rodrigues et al. (2014) contra cepas de bactérias do gênero gram-positivas; Souza et al. (2010) frente bactérias gram-positivas e gram-negativas, relatando menor atividade contra as cepas gram-negativas e Rocha (2012) demonstrando a eficácia dessa espécie contra Staphylococcus aureus. Dessa forma, Moreno et al.(2006) indagam que a ausência de formação de halo de inibição em ensaios antimicrobianos com extratos de plantas não significa necessariamente que os extratos sejam inativos frente ao microrganismo ensaiado, mas que a difusã o dos compostos não foi completada.

Dentro dessa perspectiva Vanden e Vlietinck enfatizam que mé todos microbiológicos de detecção de atividade antimicrobiana encontrados na literatura tanto para detecção da atividade de fungos como bactérias podem gerar discrepâncias nos resultados obtidos em pesquisas, pois a variedade de métodos (método de difusão em ágar por poço, disco-difusão e métodos de macrodiluiçã o e microdiluição em caldo) encontrada para esse efeito não são todos baseados no mesmo princípio. Desta maneira justificam que os resultados obtidos serão também profundamente influenciados, não só pelo método escolhido, mas também pelos microrganismos utilizados para realizar o teste, e pelo grau de solubilidade de cada teste. Resumidamente, Nascimento et al. (2007) enfatizam sobre os fatores que não devem de modo algum serem negligenciados, tais como: a técnica usada, o meio de cultura, densidade do inó culo e o emulsificador utilizado.
Diante disso, Silveira et al. (2009) ao analisarem a eficácia de dois métodos para avaliação antimicrobiana em ágar, disco-difusão e difusão em poço, concluem que a técnica do poço se apresentou mais sensível para determinação antimicrobiana de extratos vegetais que a técnica do disco utilizada neste estudo. Entretanto, os resultados apesar de mostrar melhor efetividade em termos de tamanho de halo de inibição para a técnica de poço difusão, não prioriza o emprego desta nas avaliações de extratos de produtos naturais, ou mesmo invalida o emprego da metodologia de disco difusão pela sua menor difusibilidade, esclarecem os autores. Seguindo a discursão argumentam que estas técnicas são de difícil padronização em se tratando de avaliação de produtos naturais, uma vez que existe a possibilidade de interferência devida, sobretudo, à característica do material a ser trabalhado, o que tornam os resultados disponíveis na literatura, difíceis de comparar.

Nesse intuito, Ostrosky et al. (2008) ressaltam sobre a importância de avaliar os fatores interferentes em ensaios antimicrobianos, estabelecendo parâmetros de acordo com a necessidade do método empregado para o determinado fim.

Nesse contexto, outros pesquisadores acrescentam sobre os fatores que podem influenciar a ação antimicrobiana de substâncias de origem vegetal e interferir nos resultados obtidos em ensaios microbiológicos na determinação de susceptibilidade in vitro, como as diferentes formas de preparo ou apresentação do material vegetal, seja na forma de óleo essencial, extrato (etanólico, aquoso, hidroalcoólico), além dos fatores ambientais que podem influenciar na biossíntese dos metabólitos secundários, como clima, tipo de solo, época de coleta, entre outros (FILHO; YUNES, 1997; JÚNIOR; VIZZOTTO, 1996; VANHAELEN et al., 1996). Em fase disso, o estudo conduzido por Rodrigues et al.(2014) corrobora quando de acordo com esses autores a presença de cumarinas e alcaloides foi detectada apenas no extrato alcoólico e os depesí deos e depsidonas apenas presentes no extrato aquoso.

Seguindo a discussão acima, em um outro trabalho realizado por Tadeg et al. (2005), utilizando o método de difusão em ágar, os melhores resultados foram obtidos para as frações clorofórmio e é ter de petróleo de Lippia adoensis contra bactérias e Olinia rachetiana contra fungos. Assim, o uso de solventes distintos resulta na extração diferenciada de componentes bioativos, conforme a polaridade de ambos, exercendo função terapêutica segundo o tipo de composto extraído. Sendo, portanto, o tipo de solvente um dos fatores que devem ser considerados, podendo desta maneira, inferir na susceptibilidade dos agentes antimicrobianos de origem vegetal.

A figura 1 mostra o resultado da análise antifúngica utilizando a maior concentração do estudo que foi de $100 \mathrm{mg}$ do extrato etanó lico das cascas de $A$. macrocarpa. 0 ensaio evidencia que o extrato foi inativo ao microrganismo testado, não havendo formação de zonas de inibição de crescimento microbiano na presença do extrato vegetal na concentração teste.

Ainda em relação ao ensaio representado na figura 1 é possível notar a presença de resíduos de extratos adjacente ao disco de papel filtro, refletindo na baixa difusibilidade dos possíveis agentes antimicrobianos presentes no extrato. É nesse sentido que Bona et al. (2014) questionam a desvantagem desse método em relação aos resquícios de amostra vegetal que permanecem aderidos ao disco após a difusão em ágar no que se refere a sua influência na mediçã o dos halos e na concentração do próprio disco.

Somado ao que foi supracitado, Estrela (2000) discorre que o mé todo de difusão em ágar não oferece condições de igualdade para se comparar substâncias com solubilidade e difusibilidade distintas. Dados do estudo desenvolvido por este autor sustentam que algumas substâncias apresentam dificuldades de difusão e dissociaç ão em ágar. 


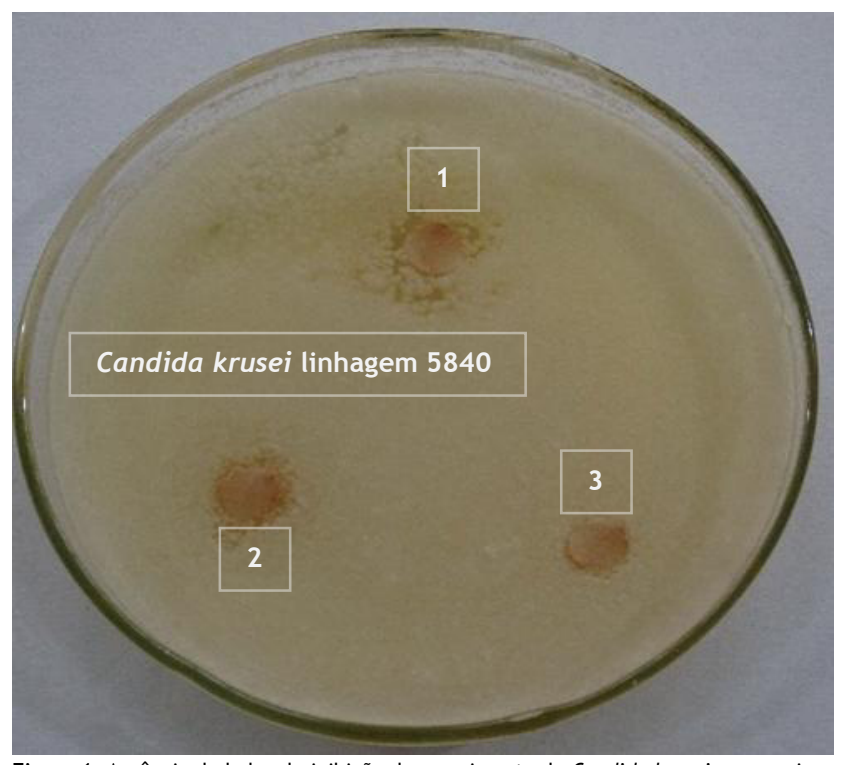

Figura 1. Ausência de halos de inibição de crescimento de Candida krusei em ensaio com o extrato etanólico de Anadenanthera macrocarpa na concentração de $100 \mathrm{mg}$ em triplicata $(1,2,3)$. (Fonte: produção do autor, 2017).

Pinto; kaneko e Ohara (2003) esclarecem que pode-se utilizar recursos para melhoria da leitura do diâmetro do halo no método de difusão em ágar, argumentando que a espessura e a uniformidade do ágar são essenciais para a boa resolução dos resultados. A esse respeito, os autores afirmam que quando ocorrem halos de pequenas dimensões, o recurso utilizado é a diminuição do volume do meio, o que leva a diminuição a espessura do meio na placa, utilizando-se a pré-difusão quando se visa aumentar a zona de inibição.

$\mathrm{Na}$ avaliação dos ensaios para verificar a atividade antifúngica do controle negativo (Tabela 3 ), constatou-se que o emulsificante dimetilsulfóxido na presença da cepa de Candida krusei não provocou inibição de crescimento microbiano. Sendo assim, não houve interferência do diluente sobre a ação do extrato.

Tabela 3. Medidas dos diâmetros dos halos de inibição de crescimento microbiano $(\mathrm{mm})$ obtidas pela metodologia de difusão em ágar por disco utilizando antimicrobianos comerciais (Miconazol e Nistatina) frente Candida krusei.

\begin{tabular}{c|c}
\hline Controles positivos e negativos & $\begin{array}{c}\text { Média da zona } \\
\text { de inibição }\end{array}$ \\
\hline Miconazol $(20 \mathrm{mg} / \mathrm{g})$ & 12 \\
\hline Nistatina $(22,1 \mathrm{mg} / \mathrm{mL})$ & - \\
\hline Dimetilsulfóxido & - \\
\hline (-): ausência de zona de inibičão.
\end{tabular}

A cepa fúngica em estudo, apresentou bom espectro de sensibilidade ao antimicrobiano usual Miconazol, o que favoreceu a formação de halo de inibição consistente, à exceção da Nistatina, onde se observou resistência da cepa $C$. krusei a este antifúngico comercial (Tabela 3). Com referência a tal contexto, estudos apontam para menor efetividade desse fármaco na terapia contra espécies de patógenos causadoras da candidíase (FERRAZZA et al., 2005).

Os resultados da atividade antifúngica obtidos neste estudo são similares aos resultados encontrados por Costa et al. (2013) ao observar que o extrato etanólico de $A$. macrocarpa não apresentou resultados positivos para a ação antifúgica contra outras linhagens do gênero Candida, que são elas: $C$. albicans, $C$. tropicalis e $C$. glabrata. Contudo, não foram encontradas publicações referentes à atividade antifúngica do extrato etanólico das cascas de $A$. macrocarpa com a utilização do método de disco-difusão em ágar frente ao microrganismo referência.

\section{CONCLUSÕES}

A partir dos testes realizados, é possível concluir que a cepa padrã o ensaiada não se mostrou sensível ao extrato vegetal pela técnica de disco-difusão, sugerindo que testes complementares sejam realizados utilizando-se outros métodos de investigação de atividade antimicrobiana em base ágar para verificação dos resultados encontrados neste estudo, quanto a eficiência do extrato etanólico de $A$. macrocarpa em inibir o crescimento fú ngico da espécie Candida krusei, confirmando ou não os pressupostos levantados nesta pesquisa.

Por fim, os dados obtidos com o presente estudo equiparado aos relatos de pesquisas na literatura chamam a atenção para a necessidade de padronização da diversidade de técnicas acerca de atividade antimicrobiana em extratos de plantas, bem como as variáveis relacionadas a essas amostras que podem interferir na sua eficácia em inibir o crescimento do microrganismo em teste, e que da mesma forma devem ser levadas em consideração em ensaios desta natureza.

\section{REFERÊNCIAS}

ALFAIA, Daiani Patrícia Silva; ALMEIDA, Sheylla Susan Moreida da Silva. Avaliação fitoquímica, análise citotóxica e antimicrobiana do extrato bruto etanólico das folhas de Annona muricata L. (Annonaceae). Biota Amazônia, Macapá, v. 6, n. 1, p. 26-30, 2016. BARBEDO, Leonardo S.; SGARBI, Diana B.G.; Candidíase. DST - J. bras. Doenças Sex. Transm. v. 22, n. 1, p. 22-38, 2010.

BONA, E. A. M. et al. Comparação de métodos para avaliação da atividade antimicrobiana e determinação da concentração inibitó ria mínima (cim) de extratos vegetais aquosos e etanólicos. Arq. Inst. Biol., São Paulo, v. 81, n.3, p. 218-225, 2014.

COSTA, E.M.M.B. et al. In vitro antimicrobial activity of plant extracts of semi-arid region of Paraíba, PB, Brazil. Rev. Odonto. Cienc. v.28, n.4, p.101-104, 2013.

ESTRELA, C. R. A. Eficácia antimicrobiana de soluções irrigadoras de canais radiculares. 2000. 88f. Mestrado. Instituto de Patologia Tropical e de Saúde Pública de Goiânia, Goiás.

FERNANDES, Tamás Jataí Soares. Atividade antimicrobiana e determinação da CIM de extratos e fases particionadas de produtos vegetais. 2014. 56f. Monografia (Graduação Bacharelado em Farmácia). Universidade Estadual da Paraíba, Campina Grande, PB.

FERRAZZA, Magda Helena S. H. et al. Caracterização de leveduras isoladas da vagina e sua associação com candidíase vulvovaginal em duas cidades do sul do Brasil. Rev. Bras. Ginecol. Obstet.; v. 27, n. 2, p. 58-63, 2005.

FILHO, Valdir Cechinel; YUNES, Rosendo A. Estratégias para a obtenção de compostos farmacologicamente ativos a partir de plantas medicinais. Conceitos sobre modificação estrutural para otimização da atividade. Química Nova, v. 21, n. 1, 1998.

JÚNIOR, A. G.; GRIGOLETO; A. R. L.; FREGONEZI, P. A. G. Candidí ase vaginal: uma questão de educação em saúde. Brazilian Journal of Health, v. 2, n. 2/3, p. 89-96, Maio/Dez, 2011.

JÚNIOR, A. A. S.; VIZZOTTO, V.; Agrop. Catarinense, 1996, 9, 5. KONEMAN E.W et al. Antimicrobial susceptibility testing. In: Color atlas and text book of diagnostic microbiology. 5.ed. PhiladelphiaNew York: Lippncott, 1997.

MATOS, F. J. A. O Formulário fitoterápico do professor Dias da Rocha: informações sobre o emprego na medicina caseira, de plantas do Nordeste, especialmente do Ceará, EUFC, Fortaleza, Brazil, 1997.

MORENO, S. et al. Antioxidant and antimicrobial activities of rosemary extracts linked to their polyphenol composition. Free Radical Research, Buenos Aires, v.40, p.223-231, 2006. 
NASCIMENTO, P. F. C. et al. Atividade antimicrobiana dos óleos essenciais: uma abordagem multifatorial dos métodos. Brazilian Journal of Pharmacognosy, v. 17, n. 1, p. 108-113, Jan./Mar. 2007.

NCCLS, National Committee for Clinical Laboratory Standards. Mé todo de Referência para Testes de Diluição em Caldo para a Determinação da Sensibilidade a Terapia Antifúngica das Leveduras; Norma Aprovada-Segunda Edição. NCCLS document M27-A2 [ISBN 1-56238-469-4]. NCCLS, 940 West Valley Road, Suite 1400, Wayne, Pennsylvania 19087-1898, Estados Unidos, 2002.

OSTROSKY, Elissa A. et al. Métodos para avaliação da atividade antimicrobiana e determinação da concentração Mínima Inibitória (CMI) de plantas medicinais. Rev. Bras. Farmacogn. v. 18, n.2, Abr./Jun. 2008.

PENNA, C., Antimicrobial activity of Argentine plants used in the treatment of infectious diseases. Isolation of active compounds from Sebastiania brasiliensis. J. Ethnopharmacol. v. 77, p. 37-40, 2001.

PEREIRA, R. J.; CARDOSO, M. G. Metabólitos secundários vegetais e Benefícios antioxidantes. Journal of Biotechnology and Biodiversity, v. 3, n. 4, p. 146-152, nov. 2012.

PINTO, T.J.A.; KANEKO, T.M.; OHARA, M.T. Controle Biológico de Qualidade de Produtos Farmacêuticos, Correlatos e Cosméticos. 2.ed. São Paulo: Atheneu Editora, 325 p, 2003.

ROCHA, E. A. L. S. S. Estudo in vitro da atividade antimicrobiana de extratos vegetais do semiárido brasileiro contra bactérias relacionadas à infecção endodôntica. 2012. 27f. Monografia (Graduação em Odontologia). Universidade Estadual da Paraíba, Campina Grande.

RODRIGUES, Laís Azevedo. Elaboração de um fitoterápico antibió tico a partir da casca de Anadenanthera macrocarpa. Revista científica da faminas - v. 10, n. 3, Set.-Dez. 2014.

SILVA, Kelle Oliveira. Avaliação das atividades antimicrobiana, aderência, antioxidante, anti inflamatória e antinociceptiva de anadenanthera macrocarpa (Benth) Brenan. 2011. 97f. Dissertaçã o (Mestrado em Ciências Fisiológicas). Universidade Federal da Bahia, Vitória da Conquista - BA.

SILVEIRA, L. M. S. et al. Metodologias de atividade antimicrobiana aplicadas a extratos de plantas: comparação entre duas técnicas de ágar difusão. Rev. Bras. Farm., v. 90, n. 2, p. 124,-128, 2009.

SOUZA, L. B. F. C. Avaliação da atividade antimicrobiana do extrato do Sanativo ${ }^{\circledR}$ em bactérias gram-positivas e gramnegativas. Ciências Biológicas - 10. Microbiologia - 2. Microbiologia aplicada. Reunião Anual da SBPC, 62., Natal, 2010.

TADEG, $H$. Antimicrobial activities of some selected traditional Ethiopian medicinal plants used in treatment of skin disorders. J. Ethnopharmacol, v.100, p.168-175, 2005.

TORTORA, G. J.; FUNKE, B. R.; CASE, C. L. Doenças Fúngicas do Sistema Reprodutor. In: TORTORA, G. J.; FUNKE, B. R.; CASE, C. L. Microbiologia. $10^{\text {a }}$ ed. Porto Alegre: Artmed, 2012.

VANDEN B. D. A.; VLIETINCK, A. J., p.47.69. Screening methods for antibacterial and antiviral agents from higher plants. In: DEY, P.M. \& HARBONE, J.D. (eds), Methods in Plant Biochemistry, Academic Press, London, 1991.

VANHAELEN, M. et al., In The Medicinal Plant Industry; Wijesekera, R. O. B.; CRC Press, Inc., Boca Raton, USA 1991, p. 59. VOLPATO, A. M. M. Avaliação do potencial antibacteriano de Calendula officinalis (Asteraceae) para seu emprego como fitoterápico. 2005. 137f. Tese (Doutorado em Ciências, concentraç ão em Química Orgânica). Universidade Federal do Paraná, Curitiba, 2005. 